\title{
Preventive Effects of Quercetin against the Onset of Atherosclerosis-Related Acute Aortic Syndromes in Mice
}

\author{
Masateru Kondo $^{1,2}$, Yuki Izawa-Ishizawa ${ }^{3, *} \mathbb{C}$, Mitsuhiro Goda ${ }^{2}{ }^{(0}$, Mayuko Hosooka ${ }^{4}$, \\ Yuu Kagimoto ${ }^{1}$, Naoko Saito ${ }^{5}$, Rie Matsuoka ${ }^{1,2}{ }^{\text {, }}$ Yoshito Zamami ${ }^{1}$, Masayuki Chuma ${ }^{6}$, \\ Kenta Yagi ${ }^{6}{ }^{D}$, Kenshi Takechi ${ }^{7}$, Koichi Tsuneyama ${ }^{8}$ and Keisuke Ishizawa ${ }^{1,2}$ \\ 1 Department of Clinical Pharmacology and Therapeutics, Tokushima University Graduate School of \\ Biomedical Sciences, Tokushima 770-8503, Japan; kondou.masateru@tokushima-u.ac.jp (M.K.); \\ uk.exile-namtk511@hotmail.co.jp (Y.K.); r.matsuoka@tokushima-u.ac.jp (R.M.); \\ zamami@tokushima-u.ac.jp (Y.Z.); ishizawa@tokushima-u.ac.jp (K.I.) \\ 2 Department of Pharmacy, Tokushima University Hospital, Tokushima 770-8503, Japan; \\ mgoda@tokushima-u.ac.jp \\ 3 AWA Support Center, Tokushima University, Tokushima 770-8503, Japan \\ 4 Department of Medical Pharmacology, Tokushima University Graduate School of Biomedical Sciences, \\ Tokushima 770-8503, Japan; mayu7015@hotmail.co.jp \\ 5 Department of Pharmacology, Tokushima University Graduate School of Biomedical Sciences, \\ Tokushima 770-8503, Japan; sf3ft5ym7ys9nm@gmail.com \\ 6 Clinical Research Center for Developmental Therapeutics, Tokushima University Hospital, \\ Tokushima 770-8503, Japan; chuma.masayuki@tokushima-u.ac.jp (M.C.); \\ yagi.kenta@tokushima-u.ac.jp (K.Y.) \\ 7 Department of Clinical Pharmacy, College of Pharmaceutical Sciences, Matsuyama University, \\ Ehime 790-8578, Japan; k.takechi@g.matsuyama-u.ac.jp \\ 8 Department of Pathology and Laboratory Medicine, Tokushima University Graduate School of Biomedical \\ Sciences, Tokushima 770-8503, Japan; tsuneyama.koichi@tokushima-u.ac.jp \\ * Correspondence: ishizawa.yuki@tokushima-u.ac.jp; Tel.: +81-88-633-7538
}

Received: 2 September 2020; Accepted: 29 September 2020; Published: 30 September 2020

\begin{abstract}
Atherosclerosis-related acute aortic syndromes, such as aortic aneurysms or aortic dissection are life-threatening diseases. Since they develop suddenly and progress rapidly, the establishment of preventive strategies is urgently needed. Quercetin, a flavonoid abundant in various vegetables and fruits, is suggested to reduce the risk of cardiovascular disease. Therefore, in this study, the preventive effect of quercetin was evaluated using a mouse model of aortic aneurysm and dissection. The model was established by administering angiotensin II (Ang II) and $\beta$-aminopropionitrile (BAPN), a lysyl oxidase inhibitor, to mice to induce hypertension and degeneration of the elastic lamina, which would eventually result in the onset of an aortic aneurysm. Ang II, BAPN, and a nitric oxide synthase inhibitor was administered to induce aortic dissection via endothelial dysfunction. Quercetin $(60 \mathrm{mg} / \mathrm{kg} /$ day $)$ was administered 2 weeks before inducing aortic diseases by the end of the experiments ( 8 weeks in the aneurysm model, 6 weeks in the dissection model). It was found to reduce the incidence of aneurysm (from 72 to $45 \%$ ), dissection (from 17 to $10 \%$ ), and rupture (from 33 to $15 \%$ ) in mice. Elastin degradation was ameliorated in the quercetin-treated mice compared to that in the mice without quercetin treatment (degradation score $2.9 \pm 0.3$ vs $2.2 \pm 0.2$ ). Furthermore, quercetin suppressed the expression of vascular cell adhesion molecule-1, macrophage infiltration, and pro-matrix metalloproteinase- 9 activity. Our results suggest that quercetin might prevent the onset of atherosclerosis-related acute aortic syndromes through its anti-inflammatory and endothelial cell-protective effects.
\end{abstract}

Keywords: flavonoids; aortic aneurysm; aortic dissection; endothelial dysfunction; inflammation 


\section{Introduction}

Aortic aneurysms and aortic dissection are among the atherosclerosis-related acute aortic syndromes [1-3]. An aneurysm is a condition in which a part of the wall of the aorta circumferentially or locally enlarges or protrudes [4]. The known causes of aortic aneurysms are atherosclerosis, hypertension, and weakening of elastic lamina. An ultrasonic screening study revealed the prevalence of abdominal aortic aneurysms among individuals aged 65 years and higher is $4-7 \%$ in men and $1-2 \%$ in women [5]. In most cases, patients are asymptomatic initially but have a fatal outcome once the aneurysm ruptures [4]. Thus far, only antihypertensive agents have been used therapeutically against aortic aneurysm enlargement or aortic rupture [4].

Aortic dissection is a condition in which the aortic wall is separated into two layers at the level of the media, resulting in the formation of a true lumen and false lumen [4]. Although the cause of dissection is considered to be similar to that of aneurysms, endothelial dysfunction also plays an important role in the onset of dissection [6,7]. In our previous study, we established a novel aortic dissection-prone model mouse that is pharmacologically induced by loading angiotensin II (Ang II) and $\beta$-aminopropionitrile (BAPN), a lysyl oxidase inhibitor, to cause hypertension and degeneration of the elastic lamina, respectively, resulting in the onset of an aortic aneurysm [8]. The addition of $N \omega$-nitro-L-arginine methyl ester (L-NAME), a nitric oxide synthase (NOS) inhibitor, to this aneurysm model increases the incidence of aortic dissection and aortic rupture due to endothelial dysfunction [6]. We have previously reported that the lipid-lowering drug pitavastatin can inhibit the onset of aortic dissection through its endothelium-protecting and anti-inflammatory effects [6].

Both aortic dissection and aortic rupture due to aneurysms develop extremely rapidly and are associated with a high probability of death [9]. Therefore, we consider that the most important and effective strategy against these atherosclerosis-related acute aortic syndromes is to establish preventive measures that can be effective before the onset of diseases. Several epidemiological studies have shown that a healthy flavonoid-rich diet could help prevent cardiovascular diseases [10,11].

Quercetin, a flavonoid abundant in vegetables and fruits, such as onions, shows a potent antioxidative effect [12]. As with other flavonoids, quercetin is thought to be protective against cardiovascular diseases through its endothelium-protecting and anti-inflammatory effects $[13,14]$. It is considered that most quercetin exists in the plasma as a metabolite that has undergone glucuronidation or sulfate conjugation. Among these metabolites, quercetin-3-O- $\beta$-D-glucuronide (Q3GA) has been reported to have antioxidative effects similar to those of quercetin [15]. In our previous study, we reported that Q3GA inhibits vascular smooth muscle cell proliferation and migration via its inhibitory effects on mitogen-activated protein (MAP) kinase activity in vitro [16]. In the present study, we examined the preventive effects of quercetin, for in vivo experiments, or Q3GA, for in vitro experiments, on the onset of aortic aneurysm or dissection.

\section{Results}

The preventive effects of quercetin against aortic aneurysm or dissection were examined using two different mouse models, which were designated as $\mathrm{AB}$, by taking the acronym of two administered drugs; angiotensin II (Ang II) $+\beta$-aminopropionitrile (BAPN)-treated aortic aneurysm model mice, and $\mathrm{LAB}$, by taking the acronym of three administered drugs; N $\omega$-nitro-L-arginine methyl ester (L-NAME) + Ang II + BAPN-treated aortic dissection model mice.

\subsection{Quercetin Suppresses Aortic Aneurysm Onset via Anti-Inflammatory Effects}

Quercetin treatment was performed for 8 weeks from 2 weeks before the induction of an aneurysm until the end of the experiments. Quercetin treatment did not affect body weight and systolic blood pressure compared to the $\mathrm{AB}$ group without quercetin throughout the experimental period (Figure 1a,b). As shown in Figure 1c, more aortas from the quercetin-treated mice showed normal vasculature compared to the aortas from $\mathrm{AB}$ mice. Quercetin significantly suppressed the enlargement 
of the abdominal aortic diameter (Figure 1e) and reduced the incidence of aortic aneurysms (from $72 \%$ in the $\mathrm{AB}$ group to $45 \%$ in the quercetin group) and death from rupture (from 33 to $15 \%$ ) (Table 1). Survival rate was significantly improved in the quercetin-treated group (Figure 1f).

(a)

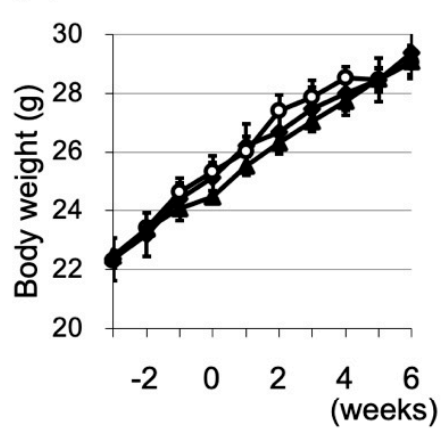

(b)

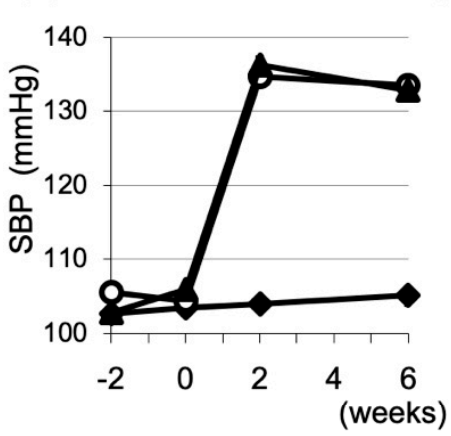

(c)

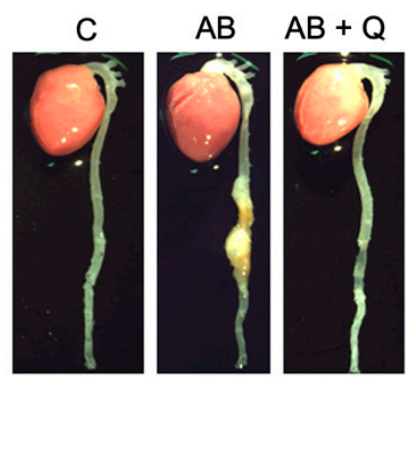

(d)

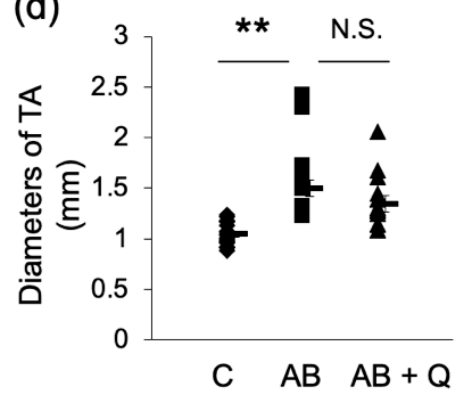

(e)

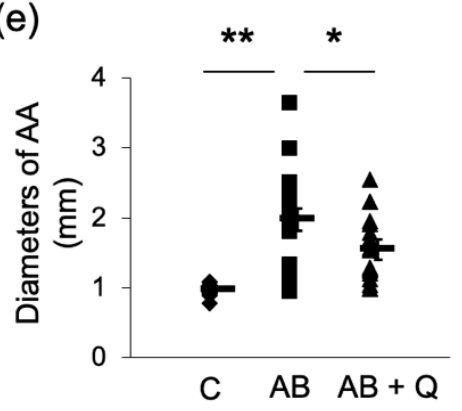

(f)

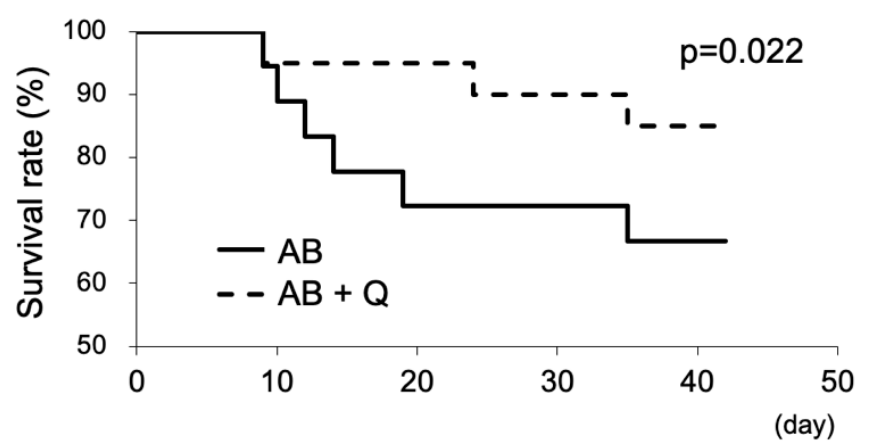

Figure 1. The effects of quercetin administration on aneurysm model mice. $C$, control; $A B$, angiotensin II (Ang II) + $\beta$-aminopropionitrile (BAPN) treated group; $Q$, quercetin; $\mathrm{SBP}$, systolic blood pressure; TA, thoracic aorta; AA, abdominal aorta. Week 0 or day 0 indicate the starting day of Ang II plus BAPN loading. The values of body weight (a) and SBP (b) were mean \pm S.E. (solid diamond: control, open circle: $\mathrm{AB}$ group, solid triangle: $\mathrm{AB}+\mathrm{Q}$ group). Panel (c) shows representative appearance of aortas. The maximum diameters of TA (d) and AA (e) are shown. Solid bars show means \pm S.E. $n=18-20$. Values are statistically analyzed using two-way analysis of variance (ANOVA) for repeated measures $(\mathbf{a}, \mathbf{b}, \mathbf{d}, \mathbf{e}) .{ }^{*} p<0.05,{ }^{* *} p<0.01$. (f) Survival rates were analyzed by Kaplan-Meier method. 
Table 1. Incidence of aortic aneurysms and rupture.

\begin{tabular}{ccccc}
\hline & $\begin{array}{c}\text { Control, } \boldsymbol{n} \\
\mathbf{( \% )}\end{array}$ & $\mathbf{A B}, \boldsymbol{n} \mathbf{( \% )}$ & $\mathbf{A B}+\mathbf{Q}, \boldsymbol{n} \mathbf{( \% )}$ & $\boldsymbol{p}$ Value * \\
\hline TAA & $0 / 18(0 \%)$ & $3 / 18(17 \%)$ & $2 / 20(10 \%)$ & 0.653 \\
AAA & $0 / 18(0 \%)$ & $13 / 18(72 \%)$ & $9 / 20(45 \%)$ & 0.090 \\
total aneurysm & $0 / 18(0 \%)$ & $13 / 18(72 \%)$ & $9 / 20(45 \%)$ & 0.090 \\
rupture & $0 / 18(0 \%)$ & $6 / 18(33 \%)$ & $3 / 20(15 \%)$ & 0.26 \\
\hline
\end{tabular}

* AB vs. AB + Q by Fisher's exact test or Pearson's chi-square test. Abbreviations are as below: AB, Ang II + BAPN; $\mathrm{Q}$, quercetin; TAA, thoracic aortic aneurysm; AAA, abdominal aortic aneurysm.

The effects of quercetin on the degeneration and collapse of elastic laminae in the aortic aneurysm model mice were assessed based on the degradation score under Elastic-Van Gieson (EVG) staining (Figure 2a). Compared to the control group (average score: $1.0 \pm 0.0$ ), the AB group showed enhanced elastin degradation (average score: $2.9 \pm 0.3$ ), which was significantly suppressed by quercetin administration (average score: $2.2 \pm 0.2$ ) (Figure $2 b$ ). The activity of pro-matrix metalloproteinase (MMP)-9, which is abundant in macrophages, was significantly inhibited by quercetin in the aorta compared to that in the $\mathrm{AB}$ group (Figure $2 \mathrm{c}, \mathrm{d}$ ). On the other hand, there was no significant difference in the activity of MMP-2 and pro-MMP-2, which is abundant in vascular smooth muscle cells (Figure 2e,f).

(a)

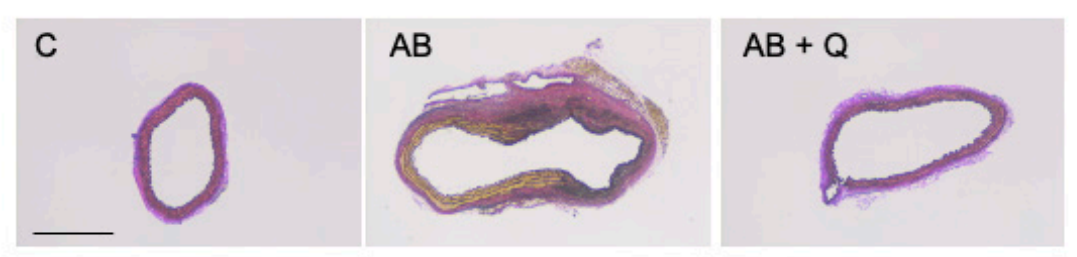

(b)

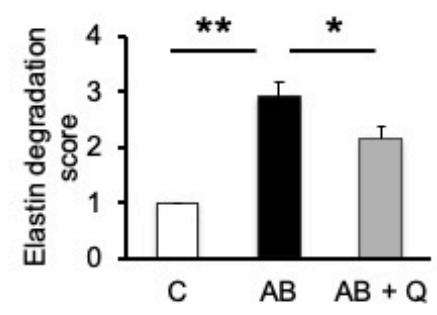

(c)

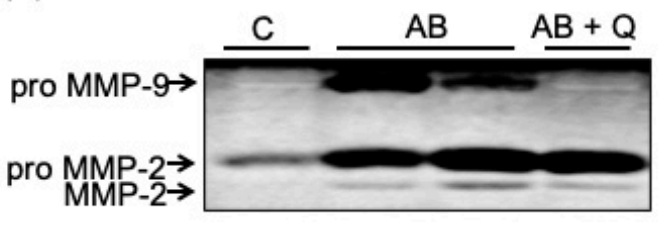

(d)

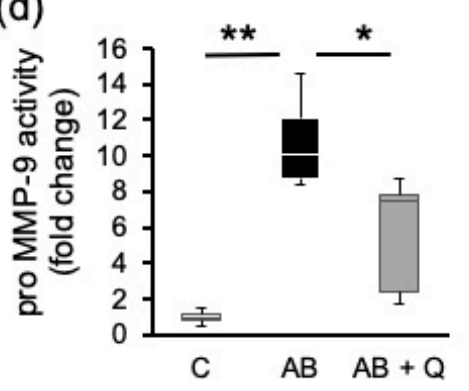

(e)

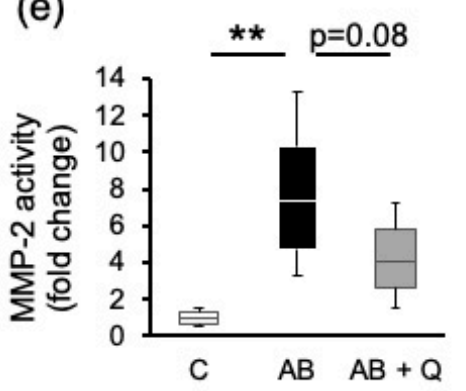

(f)

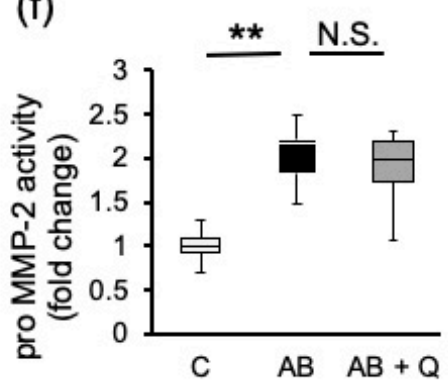

Figure 2. The effects of quercetin on elastin degradation. (a) The representative pictures of Elastic- Van Gieson (EVG) staining (bar: $0.5 \mathrm{~mm}$ ). (b) The average values of elastin degradation score estimated under EVG staining $(n=13-20)$. (c) The representative figures of zymography assay. Quantified intensity of each band is shown in (d), (e), and (f) $(n=4-8)$. Values are shown as fold increase to the average of control and expressed as mean \pm SE. Statistical analyses were performed using two-way ANOVA for repeated measures and Bonferroni post hoc test. ${ }^{*} p<0.05,{ }^{* *} p<0.01$. AB, Ang II + BAPN; $\mathrm{Q}$, quercetin; MMP, matrix metalloproteinase. 
Furthermore, quercetin suppressed macrophage infiltration into the aortic wall, as indicated by the immunostaining of Mac-2 (Figure 3a) and mRNA expression of F4/80 (Figure 3b). These findings were consistent with the results that pro-MMP-9 was suppressed in quercetin treated aortas, as shown in Figure $2 \mathrm{~d}$. The expression of vascular cell adhesion molecule (VCAM)-1, which can recruit inflammatory cells including macrophages, was also suppressed by quercetin treatment (Figure 3c).

(a)

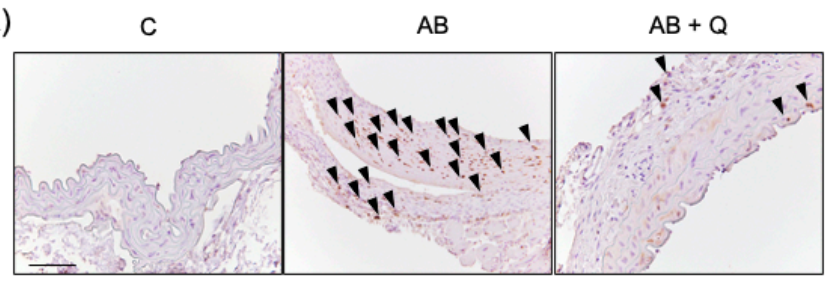

(b)

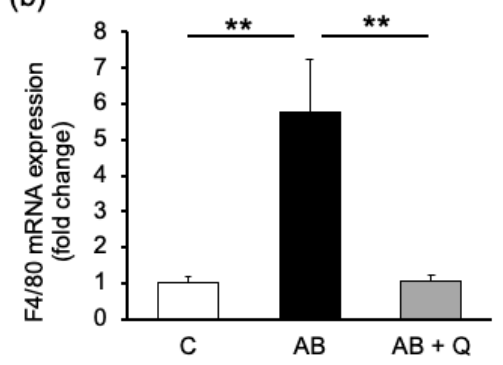

(c)

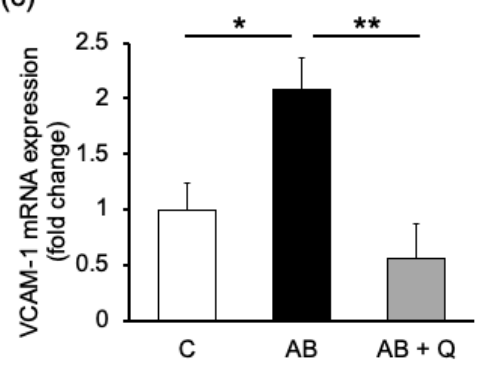

Figure 3. The effects of quercetin on inflammatory responses in aortas. Panel (a) shows representative pictures of Mac- 2 expressions. Arrow heads are positive staining. Bar: $0.1 \mathrm{~mm}$. Graphs show the mRNA expressions of inflammatory markers, F4/80 (b) and vascular cell adhesion molecule (VCAM)-1 (c) in aortas. $n=4-6$. Values are shown as fold increase to the average of control and are expressed as mean \pm SE. Statistical analyses were performed using two-way ANOVA for repeated measures and Bonferroni post hoc test. ${ }^{*} p<0.05,{ }^{* *} p<0.01$. AB, Ang II + BAPN; Q, quercetin.

\subsection{Quercetin Shows Endothelial Cell-Protective Effects in Cultured Human Umbilical Vein Endothelial Cells} (HUVECS)

Tumor necrosis factor (TNF)- $\alpha$, an inflammatory cytokine, potently induces VCAM-1 [17]. TNF- $\alpha$ in mouse plasma was significantly increased in the AB group (average: $2.79 \mathrm{pg} / \mathrm{mL}$ ) as compared to the control group (average: $0.42 \mathrm{pg} / \mathrm{mL}$ ). There was no difference between the $\mathrm{AB}$ group and quercetin-treated group (average: $2.35 \mathrm{pg} / \mathrm{mL}$ ) (Figure 4a). Therefore, we examined the effect of quercetin on TNF- $\alpha$-induced signaling in cultured human umbilical vein endothelial cells (HUVECs), which is well established for research on TNF- $\alpha$ signaling to induce VCAM-1 expression $[17,18]$, using quercetin-3-O- $\beta$-D-glucuronide (Q3GA), a quercetin metabolite. VCAM-1 expression was increased by TNF- $\alpha$ stimulation and suppressed both by quercetin and Q3GA (Figure $4 b$ ). Endothelial NOS (eNOS), which is an important molecule for maintaining endothelial cell function, was downregulated by TNF- $\alpha$ stimulation, but recovered by Q3GA pretreatment (Figure $4 \mathrm{~d}$ ). Moreover, Q3GA, as well as pitavastatin, a positive control, phosphorylated extracellular signal-regulated kinase (ERK) 5, which is known as an endothelial cell-protective molecule and induces eNOS expression [19] in HUVECs (Figure 4c). 
(a)

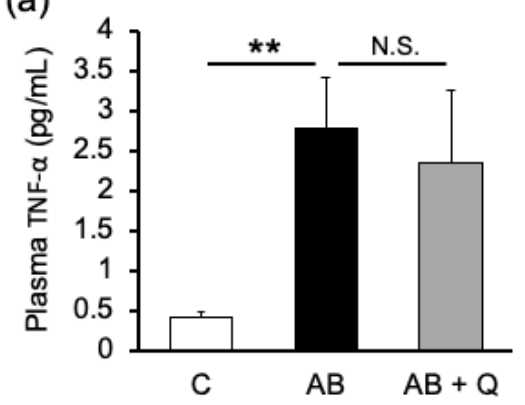

(d)
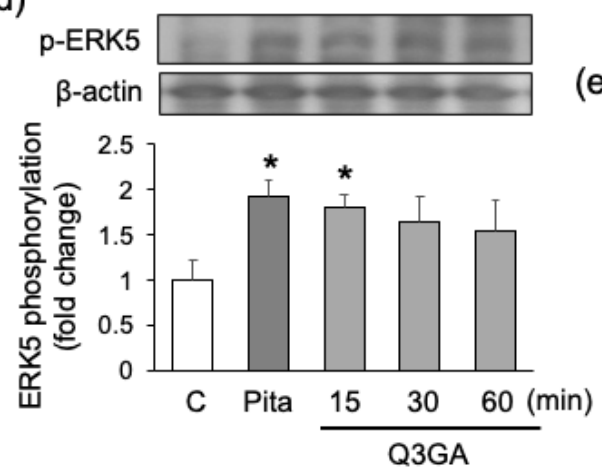

(b)

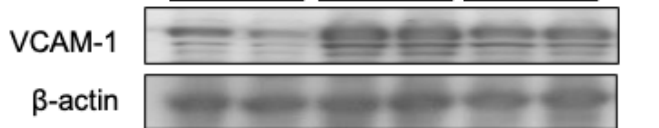

(c)

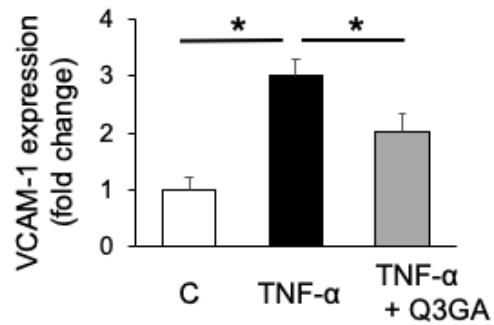

(e)

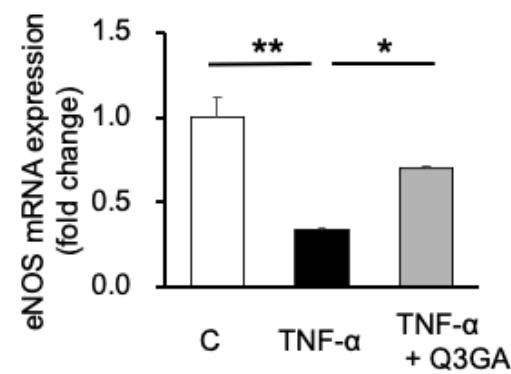

Figure 4. The effects of quercetin on plasma tumor necrosis factor (TNF)- $\alpha$ concentration and the quercetin-3-O- $\beta$-D-glucuronide (Q3GA) effects in cultured human umbilical vein endothelial cells (HUVECs). (a) TNF- $\alpha$ concentration in plasma from AB group mice $(n=5-8)$. Protein expression of VCAM-1 and extracellular signal-regulated kinase (ERK) 5 phosphorylation in HUVECs were analyzed by Western blotting. Representative bands ((b) and upper panel of (d)), and quantified intensity ((c) and lower panel of (d)) are shown. The mRNA expression of endothelial nitric oxide synthase (eNOS) in HUVEC are shown in (e). $n=4-6$. Values are shown as fold increase to the average of control and are expressed as mean \pm SE. Statistical analyses were performed using two-way ANOVA for repeated measures and Bonferroni post hoc test. ${ }^{*} p<0.05,{ }^{* *} p<0.01$ (vs. control in (d)). AB, Ang II + BAPN; Q, quercetin; Pita, pitavastatin.

\subsection{Quercetin Suppresses the Incidence of Aortic Dissection and Mortality in Mouse Models of Dissection}

Since the effect of quercetin on endothelial dysfunction was observed (Figure 4), it was hypothesized that quercetin is more effective against aortic dissection, which is highly associated with endothelial dysfunction. The effect of quercetin in the aortic dissection model (LAB group) was examined. The effect of quercetin on the incidence of aortic dissection or rupture was assessed 1 week after Ang II + BAPN loading. Figure 5a,b show representative pictures of macroscopic figures and EVG staining of mouse aortas, respectively. The incidence of aortic dissection reduced to $16 \%$ in the quercetin-treated group, compared to 33\% in the LAB group. Twenty-two percent of the mice in the LAB group died from aortic rupture, whereas none died in the quercetin-treated group (Table 2).

The effect of quercetin on the inflammatory response in the aortic dissection model was examined. Macrophage infiltration into the aortic wall, which was assessed by immunostaining of F4/80, increased in the LAB group and was suppressed by quercetin treatment (Figure $5 c, d$ ). VCAM- 1 expression in the aorta was also upregulated in the LAB group and was suppressed by quercetin treatment (Figure 5e,f). 
(a) C

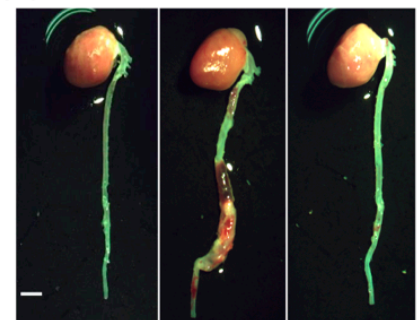

(c)

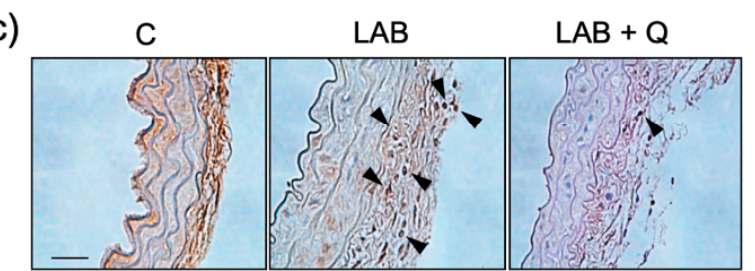

(e)

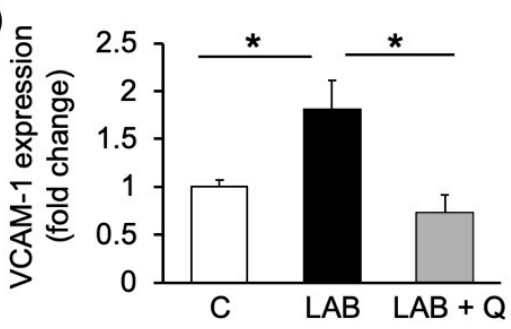

(b)

C

(f)
LAB

$\mathrm{LAB}+\mathrm{Q}$

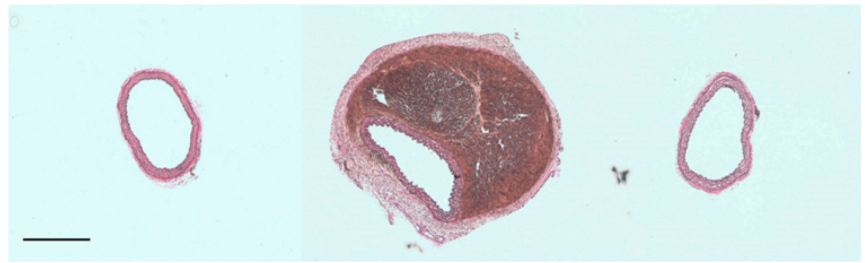

(d)
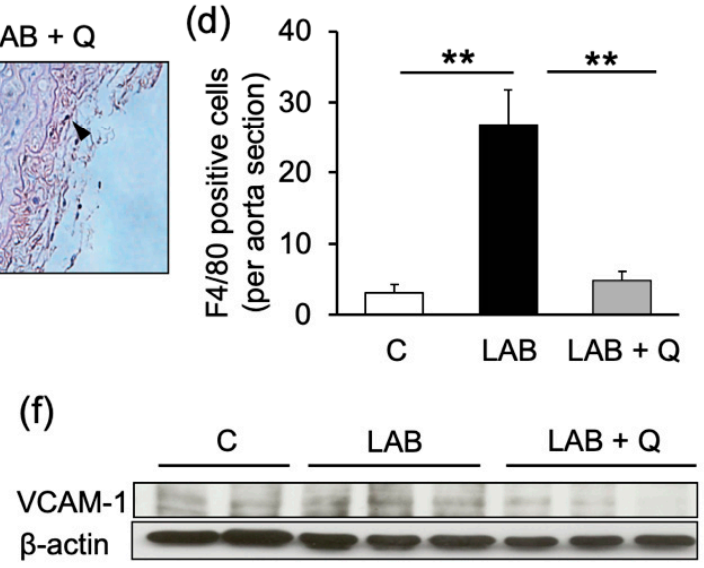

Figure 5. The effects of quercetin on aortic dissection model. The representative pictures of gross aortas (a, bar: $1.0 \mathrm{~mm}$ ) and EVG staining (b, bar: $0.5 \mathrm{~mm}$ ). The representative pictures of immunostaining by F4/80 (c, bar: $100 \mu \mathrm{m}$ ), and the quantified graph (d) are shown. Protein expression of VCAM-1 in mice aortas were analyzed by Western blotting. Representative bands (f) and quantified intensity (e) are shown. $n=4-6$. Values are shown as fold increase to the average of control and are expressed as mean \pm SE. Statistical analyses were performed using two-way ANOVA for repeated measures and Bonferroni post hoc test. ${ }^{*} p<0.05,{ }^{* *} p<0.01$. LAB, L-NAME + Ang II + BAPN; Q, quercetin.

Table 2. Incidence of aortic dissection and rupture.

\begin{tabular}{ccccc}
\hline & Control, $\boldsymbol{n} \mathbf{( \% )}$ & LAB, $\boldsymbol{n}(\mathbf{\%})$ & LAB + Q, $\boldsymbol{n}(\mathbf{\%})$ & $\boldsymbol{p}$ Value \\
\hline Dissection & $0 / 17(0 \%)$ & $6 / 18(17 \%)$ & $4 / 25(10 \%)$ & 0.275 \\
rupture & $0 / 17(0 \%)$ & $4 / 18(33 \%)$ & $0 / 25(15 \%)$ & $0.025 *$
\end{tabular}

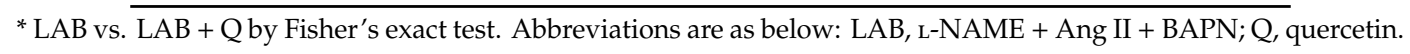

\section{Discussion}

In the present study, we suggested that quercetin administration might prevent atherosclerosisrelated acute aortic syndromes, such as aortic aneurysm, dissection, and death from rupture, in mice. The preventive effect of quercetin against these conditions was attributable to its anti-inflammatory and endothelial cell-protective effects, which were independent of its blood pressure-lowering effect.

The pathophysiological features of aortic aneurysms and dissection are very similar and are due to atherosclerosis, hypertension, and weakened elastic lamina. Since the collapse of the endothelium is essential for the onset of dissection or aortic rupture, the involvement of endothelial dysfunction is more strongly suspected in aortic dissection or rupture compared to that in aneurysms. As shown in Table 1, quercetin suppressed the incidence of aortic aneurysms from 72 to $45 \%$; however, nine out of 20 mice in the aneurysm-prone AB group still showed aneurysm formation. On the other hand, quercetin decreased the incidence of dissection and rupture from six and four out of 18 to four and zero out of 25 mice, respectively, in the dissection-prone LAB group (Table 2). In the present study, the number of animals used in the experiment was minimal; therefore, a significant difference was 
detected only in terms of a suppressive effect on aortic rupture. However, there was a clear tendency of prevention of the onset of aortic dissection. These results suggest that quercetin showed a stronger effect in the $\mathrm{LAB}$ group than in the $\mathrm{AB}$ group (Tables 1 and 2) through its endothelial protective effect.

In a previous study, we reported that an increase in nitric oxide may upregulate the expression of the cell junction molecule, vascular endothelial cadherin, via ERK5 expression and protect the endothelium from vascular hyperpermeability [6]. Pitavastatin phosphorylates ERK5 directly and activates its downstream pathways including eNOS expression [6]. In the present study, we have showed that quercetin activated ERK5 to the same extent as pitavastatin (Figure 4). Moreover, several studies have demonstrated that flavonoids such as fisetin and puerarin also activate the ERK5/MEF2c/KLF2 pathway-which induces eNOS expression-and show endothelial cell protective effects $[20,21]$. From these reports, it is strongly suggested that quercetin's effect is mediated by the ERK5 pathway. Further examination is needed to clarify the detailed molecular pathway.

Moreover, we found that quercetin could suppress VCAM-1 expression in mouse aorta and cultured HUVECs (Figures 3-5). This finding was consistent with that reported by Lee et al., showing that quercetin suppressed vascular endothelial growth factor-induced inflammatory responses, such as the translocation of nuclear factor kappa B, the upregulation of adhesion molecules and MMPs, and the downregulation of tight junction molecules [22]. In the present series of experiments, the tendency was shown that VCAM-1 expression in aorta tissue was lower in the quercetin treated sample than control sample. This tendency was observed in both mRNA expressions in aneurysm mice (Figure $3 c$ ) and protein expressions in dissection mice (Figure $5 \mathrm{e}, \mathrm{f}$ ), but not the expression in HUVECs (Figure $4 \mathrm{~b}, \mathrm{c}$ ). From these results, it was implied that sham operation or the daily administration of vehicle might have affected to basal systemic inflammatory response. Actually, other reports also showed relatively high expression of VCAM-1 in control with sham operation [23]. In the present study, quercetin was administered with high concentration, so that the expression of VCAM-1 could be suppressed to the lower level compared to the sham-treated control. Infiltrated macrophages via association with VCAM-1 play an important role in the degradation of elastic lamina through the activation of MMP-9, an extracellular matrix-degrading enzyme [24]. Since quercetin treatment significantly inhibited macrophage infiltration into the aortic wall (Figure 3), pro-MMP-9 activity also significantly decreased in the quercetin-treated mice (Figure 2).

In the present study, we demonstrated the suppressive effects on elastin degradation via the inhibition of macrophage infiltration but not via direct action to aortic media. However, we have found that quercetin also affects vascular smooth muscle cell function in the progression of cardiovascular disease in the previous study. The quercetin derivative Q3GA inhibited vascular smooth muscle cell proliferation and migration via its inhibitory effects on MAP kinase activity [16]. Pereira et al. demonstrated that quercetin decreases the activity of MMP-2 and ameliorates vascular remodeling in rats with renovascular hypertension [25]. Therefore, it is speculated that quercetin acts on both the intima and media of the aorta to suppress the onset of aortic aneurysm and dissection. Future studies need to clarify the effect of quercetin on vascular smooth muscle cells in the progression of atherosclerosis-related acute aortic syndrome.

A recent systematic review and meta-analysis on the effect of quercetin against cardiovascular diseases demonstrated that long-term supplementation with quercetin could lower blood pressure, high-density lipoprotein cholesterol, and triglycerides in humans [26]. In addition, it is very meaningful that our study showed that quercetin supplementation could prevent even sudden cardiovascular events. In this study, however, high doses of quercetin $(60 \mathrm{mg} / \mathrm{kg} /$ day, via p.o. $)$ that cannot be taken with a normal diet are administered. Therefore, the continuous intake of quercetin as a medication could be beneficial, especially for those at high risk of cardiovascular events. Further clinical investigations of the effects of quercetin on atherosclerosis-related acute aortic syndrome and application to new drug developments are desired. 


\section{Materials and Methods}

\subsection{Ethics Statement}

This study conformed to the Guide for the Care and Use of Laboratory Animals (NIH Publication No. 85-23, 1996) [27]. All animal procedures were performed in accordance with the guidelines of the Animal Research Committee of the University of Tokushima Graduate School, and the protocols were approved by the Tokushima University Institutional Review Board for animal protection; approval No. is T30-85.

\subsection{Reagents}

Angiotensin II (Ang II), $\beta$-aminopropionitrile (BAPN), Nw-nitro-L-arginine methyl ester (L-NAME), quercetin, quercetin-3-O- $\beta$-D-glucuronide (Q3GA), and tumor necrosis factor- $\alpha$ (TNF- $\alpha$ ) were purchased from Sigma-Aldrich Japan (Tokyo, Japan). $\beta$-actin (3700s) antibodies were purchased from Cell Signaling Technology Inc. (MA, USA). VCAM-1 (E-10) antibody and F4/80 (M-300) antibody were purchased from Santa Cruz Biotechnology Inc. (CA, USA). Mac2 antibody was from Cedarlane Laboratories Ltd. (NC, USA). All other reagents and instruments we used in the present study are commercially available.

\subsection{Mice and In Vivo Experimental Strategies}

C57BL/6J male mice (6-8 weeks old, weighing 20-25 g) were purchased from CLEA Japan Inc. (Tokyo, Japan). Sixty mice were used for each of the aortic aneurysm experiments and the dissection experiment in this study in accordance with statistically estimated sample sizes and divided to three groups in a random manner. The animals were housed in a temperature-controlled room at $25^{\circ} \mathrm{C}$ under a 12-h light/dark cycle with free access to food and water. C57BL/6 J mice were anesthetized by an intraperitoneal injection of $100-150 \mathrm{mg} / \mathrm{kg}$ sodium pentobarbital, and more was administered if the mice moved in response to pain after $30 \mathrm{~min}$ from the start of the operation. Pedal withdrawal reflex, toe pinch reflex, muscular relaxation, and respiratory rates were monitored to ensure that adequate anesthesia was administered. Aneurysm model mice and dissection model mice were created as previously reported [16,28]. Briefly, in the aortic aneurysm model, 11-week-old mice were implanted dorsally with two subcutaneous osmotic mini-pumps (Model 20062002 Micro-osmotic Pump; Alzet, Cupertino, CA, USA) to administer Ang II (1000 ng/kg/day for 6 weeks) and BAPN (150 mg/kg/day for 2 weeks). For development of dissection mice, L-NAME (10 mg/kg/day) was orally administered in drinking water from 8 weeks of age until the end of the experiment. After 3 weeks from the start of experiment, Ang II (1000 ng/kg/day) and BAPN (150 mg/kg/day) were administered for 1 week. Each group was abbreviated as below:

C-control, untreated group;

$\mathrm{AB}$-Ang II + BAPN treated aneurysm group;

$\mathrm{LAB}-\mathrm{L}-\mathrm{NAME}+$ Ang II + BAPN treated dissection group.

Quercetin was administered daily via a feeding needle at the dose of $60 \mathrm{mg} / \mathrm{kg} / \mathrm{day}$ based on the previous study [29] from 2 weeks before the start of other drug administration to the end of experiment; continued for 8 weeks in the aneurysm model or 6 weeks in the dissection model.

\subsection{Systolic Blood Pressure (SBP)}

SBP was measured in conscious mice by tail-cuff plethysmography (BP-98A, Softron, Tokyo, Japan).

\subsection{Tissue Sampling and Measurement of Aortic Diameter}

At the endpoint of the experiment, the animals were anesthetized by intraperitoneal injection of sodium pentobarbital (150 mg/ $\mathrm{kg}$ or more) and euthanized by cervical dislocation. The whole heart and aorta were isolated and photographed by SZ61 Olympus stereomicroscope (Olympus Corp., Tokyo, Japan). Maximum diameters of aortas were measured using ImageJ v. 1.37 software (National 
Institutes of Health, Bethesda, MD, USA). Anomalies where the abnormal site had expanded beyond 1.5-times the normal diameter were judged as aneurysms, and the aortic aneurysm incidence rate was determined. Blood samples were incubated at $37^{\circ} \mathrm{C}$ for $30 \mathrm{~min}$ and centrifuged at $2600 \times g$ for $2 \mathrm{~min}$. The supernatant serum was used to measure the TNF- $\alpha$. For the morphometry, the aortas were resected and placed in $10 \%$ buffered formalin. After fixation, the tissues were embedded in paraffin.

\subsection{Histology and Immunohistochemistry}

Sections (5 $\mu \mathrm{m}$ thickness) were subjected to Elastic-van Gieson (EVG) staining (Elastic-van Gieson staining kit; MERCK, Kenilworth, NJ, USA) according to the manufacturer's instructions. The onset of aortic dissection was determined by the formation of false lumen under EVG staining. Elastin degradation was graded as follows: grade 1, intact, well-organized elastic laminae; grade 2, elastic laminae with some interruptions and breaks; grade 3, severe elastin fragmentation or loss [28]. Immunohistochemistry was performed using Mac-2 antibody (1:200 dilution) and F4/80 antibody (1:50dilution), as previously described. Peroxidase (for Mac-2) and Mayer hematoxylin (for F4/80) were used for counterstaining. Measurements were taken using Image J v. 1.37 software.

\subsection{Quantitative Real-Time PCR}

The mRNA expression levels in the aortas or culture cells were analyzed by real-time PCR, as described previously [6]. Sequences of the amplification primer pairs are as shown in Table 3.

Table 3. Sequence of the primers used for real-time PCR.

\begin{tabular}{|c|c|c|}
\hline Target gene & Forward $\left(5^{\prime}-3^{\prime}\right)$ & Reverse $\left(5^{\prime}-3^{\prime}\right)$ \\
\hline$m F 4 / 80$ & CTTGGCTATGGGCTTCCAGTC & GCAAGGAGGACAGAGTTTATCGTG \\
\hline$m V C A M 1$ & CCATTGAAGATACCGGGAAAT & TAGCTGTCTGCTCCACAGGAT \\
\hline mpactin & AAGTGTGACGTTGACATCCG & GATCCACATCTGCTGGAAG \\
\hline hVCAM1 & GGTGGGACACAAATAAGGGTTTTGG & CTTGCAATTCTTTTACAGCCTGCC \\
\hline heNOS & TGGTACATGAGCACTGAGATCG & CCACGTTGATTTCCACTGCTG \\
\hline hractin & GCGGGAAATCGTGCGTGACATTA & ATGGAGTTGAAGGTAGTTTCGTG \\
\hline
\end{tabular}

\subsection{Zymography Assay}

MMP activities in aortic tissues were measured by zymography assay, as previously described [30]. Briefly, frozen aortas were homogenized by polytron in buffer containing $10 \mathrm{mM} \mathrm{CaCl} 2$ and $0.25 \%$ Triton-X100. Homogenates in sample buffer (250 mM Tris-HCl (pH 6.8) with $100 \mathrm{mg} / \mathrm{mL}$ SDS, 35\% grycerol, and $0.05 \%$ bromophenol blue) were subjected to electrophoresis in a $10 \%$ acrylamide gel containing $1 \mathrm{mg} / \mathrm{mL}$ gelatin. After shaking in a regeneration buffer (2.5\% Triton-X100 solution) for $1 \mathrm{~h}$, enzymatic reaction was performed in a buffer $(50 \mathrm{mM}$ Tris- $\mathrm{HCl}(\mathrm{pH} 7.6)$ with $10 \mathrm{mM} \mathrm{CaCl} 2$ and $1 \%$ Triton-X100) at $37^{\circ} \mathrm{C}$ for $20 \mathrm{~h}$. Thereafter, protein staining (Coomassie Brilliant Blue R) was performed. The intensities of bands were analyzed using ImageJ v. 1.37 software.

\subsection{ELISA for TNF- $\alpha$ Measurement}

TNF- $\alpha$ in mouse plasma was quantified using the Quantikine Mouse TNF- $\alpha$ ELISA Kit (R \& D systems, Minneapolis, MN, USA) according to the manufacturer's instructions. Briefly, sample plasma or standard peptide were reacted with Diluent Buffer at room temperature for $2 \mathrm{~h}$ and followed by the reaction with conjugate for $2 \mathrm{~h}$ and substrate solution for $30 \mathrm{~min}$ sequentially. After stopping the reaction, the absorbance was measured at $450 \mathrm{~nm}$.

\subsection{Cell Culture}

Human umbilical vein endothelial cells (HUVECs) were purchased from Lonza Japan Inc., which confirmed the cell quality by the expressions of von Willebrand factor, Factor VIII, CD31 and CD105. 
All cells were cultured in Endothelial Cell Growth Medium (EGM)-2 Bullet Kit (Lonza Japan Inc, Tokyo, Japan) with $4 \%$ fetal bovine serum. Passage 4-6 cells were grown to confluence and treated with the quercetin derivatives Q3GA or TNF- $\alpha$ for indicated time and concentrations. Cell lysates were subjected to Western blotting or real time PCR analysis.

\subsection{Western Blotting}

Western blotting analysis for protein expression and ERK5 phosphorylation in mice aortas or HUVECs was performed as described previously [6]. Briefly, lysates of aortas or HUVECs were subjected to sodium dodecyl sulphate-polyacrylamide gel electrophoresis and electro-transferred to polyvinylidene difluoride membranes, as described previously. The membranes were blocked in phosphate-buffered saline with $0.1 \%$ Tween-20 (PBS-T), containing $4 \%$ milk powder for $90 \mathrm{~min}$ at room temperature and then incubated overnight at $4{ }^{\circ} \mathrm{C}$ with one of the following primary antibodies: VCAM-1 antibody (sc-8304; Santa Cruz, Santa Cruz, CA, USA), phospho-ERK5 antibody (\#3371; Cell Signaling, Danvers, MA, USA), ERK5 antibody (\#3372; Cell Signaling), or $\beta$-actin antibody (as a loading control, \#3700; Cell Signaling). The membranes were subsequently incubated with horseradish peroxidase-conjugated anti-rabbit IgG (GE Healthcare Biosciences, Piscataway, NJ, USA) for $1 \mathrm{~h}$ at room temperature, and the signals were developed using ECL Plus Western Blotting Detection System (GE Healthcare Biosciences, Piscataway, NJ, USA). Immunoreactive bands were quantified by densitometry in the linear range of film exposure using a UMAX Astra 2200 scanner and image J v. 1.37 software (National Institutes of Health, Bethesda, MD, USA).

\subsection{Statistical Analysis}

The results of the experiments were presented as means \pm standard error (SE). Statistical analysis was performed using StatMate IV for Windows (Atoms, JAPAN). The normally distributed continuous variables for more than 3 groups were compared using two-way ANOVA and for 2 groups were compared using $t$-test. Non-normal distribution continuous variables were compared using Kruskal-Wallis test and further paired comparisons were done using Mann-Whitney U test. Nominal categorical data between the groups were compared using Chi-squared $(\chi 2)$ or Fisher's exact test as appropriate. For all statistical tests, a $P$ value less than 0.05 and 0.01 was taken to indicate a significant and highly significant difference, respectively.

Author Contributions: Conceptualization, Y.I.-I.; data curation, Y.I.-I.; methodology, M.K., Y.I-I., M.H., Y.K. and N.S.; validation, M.G. and R.M.; formal analysis, K.Y. and K.T. (Kenshi Takechi); investigation, M.K., M.H., Y.K., N.S. and K.T. (Koichi Tsuneyama); resources, Y.Z. and M.C.; writing-original draft preparation, M.K.; writing-review and editing, Y.I.-I.; visualization, M.K., Y.I.-I., M.G. and R.M.; supervision, Y.I.-I. and K.I.; funding acquisition, Y.I.-I. and K.I. All authors have read and agreed to the published version of the manuscript.

Funding: This work was supported by grants from JSPS KAKENHI Grant Number, JP16K08549 (Y.I.-I.), and JP 18K06686 (K.I.).

Acknowledgments: We acknowledge expert assistance by Support Center for Advanced Medical Sciences, Institute of Biomedical Sciences, Tokushima University Graduate School. English writing support was provided by Editage of Cactus Communications K.K. (Tokyo, Japan).

Conflicts of Interest: The authors declare no conflict of interest.

\section{References}

1. Komatsu, S.; Takahashi, S.; Yutani, C.; Ohara, T.; Takewa, M.; Hirayama, A.; Kodama, K. Spontaneous ruptured aortic plaque and injuries: Insights for aging and acute aortic syndrome from non-obstructive general angioscopy. J. Cardiol. 2020, 75, 344-351. [CrossRef] [PubMed]

2. Bonaca, M.P.; O'Gara, P.T. Diagnosis and management of acute aortic syndromes: Dissection, intramural hematoma, and penetrating aortic ulcer. Curr. Cardiol. Rep. 2014, 16, 536. [CrossRef] [PubMed] 
3. Gryszczynska, B.; Budzyn, M.; Formanowicz, D.; Wanic-Kossowska, M.; Formanowicz, P.; Majewski, W.; Iskra, M.; Kasprzak, M.P. Selected atherosclerosis-related diseases may differentially affect the relationship between plasma advanced glycation end products, receptor sRAGE, and uric acid. J. Clin. Med. 2020, 9, 1416. [CrossRef] [PubMed]

4. Group, J.C.S.J.W. Guidelines for diagnosis and treatment of aortic aneurysm and aortic dissection (JCS 2011): Digest version. Circ. J. 2013, 77, 789-828. [CrossRef]

5. Eckstein, H.H.; Bockler, D.; Flessenkamper, I.; Schmitz-Rixen, T.; Debus, S.; Lang, W. Ultrasonographic screening for the detection of abdominal aortic aneurysms. Dtsch. Arztebl. Int. 2009, 106, 657-663. [CrossRef]

6. Izawa-Ishizawa, Y.; Imanishi, M.; Zamami, Y.; Toya, H.; Nagao, T.; Morishita, M.; Tsuneyama, K.; Horinouchi, Y.; Kihira, Y.; Takechi, K.; et al. Development of a novel aortic dissection mouse model and evaluation of drug efficacy using in-vivo assays and database analyses. J. Hypertens. 2019, 37, $73-83$. [CrossRef]

7. Pan, L.; Lin, Z.; Tang, X.; Tian, J.; Zheng, Q.; Jing, J.; Xie, L.; Chen, H.; Lu, Q.; Wang, H.; et al. S-Nitrosylation of plastin-3 exacerbates thoracic aortic dissection formation via endothelial barrier dysfunction. Arterioscler Thromb. Vasc. Biol. 2020, 40, 175-188. [CrossRef]

8. Kanematsu, Y.; Kanematsu, M.; Kurihara, C.; Tsou, T.L.; Nuki, Y.; Liang, E.I.; Makino, H.; Hashimoto, T. Pharmacologically induced thoracic and abdominal aortic aneurysms in mice. Hypertension 2010, 55, 1267-1274. [CrossRef]

9. Otaki, Y.; Watanabe, T.; Konta, T.; Watanabe, M.; Fujimoto, S.; Sato, Y.; Asahi, K.; Yamagata, K.; Tsuruya, K.; Narita, I.; et al. Effect of hypertension on aortic artery disease-related mortality-3.8-year nationwide community-based prospective cohort study. Circ. J. 2018, 82, 2776-2782. [CrossRef]

10. Guo, X.; Tresserra-Rimbau, A.; Estruch, R.; Martinez-Gonzalez, M.A.; Medina-Remon, A.; Castaner, O.; Corella, D.; Salas-Salvado, J.; Lamuela-Raventos, R.M. Effects of polyphenol, measured by a biomarker of total polyphenols in urine, on cardiovascular risk factors after a long-term follow-up in the predimed study. Oxid. Med. Cell Longev. 2016, 2016, 2572606. [CrossRef]

11. Hurtado-Barroso, S.; Quifer-Rada, P.; Rinaldi de Alvarenga, J.F.; Perez-Fernandez, S.; Tresserra-Rimbau, A.; Lamuela-Raventos, R.M. Changing to a low-polyphenol diet alters vascular biomarkers in healthy men after only two weeks. Nutrients 2018, 10, 1766. [CrossRef] [PubMed]

12. Simic, A.; Manojlovic, D.; Segan, D.; Todorovic, M. Electrochemical behavior and antioxidant and prooxidant activity of natural phenolics. Molecules 2007, 12, 2327-2340. [CrossRef] [PubMed]

13. Ishizawa, K.; Yoshizumi, M.; Kawai, Y.; Terao, J.; Kihira, Y.; Ikeda, Y.; Tomita, S.; Minakuchi, K.; Tsuchiya, K.; Tamaki, T. Pharmacology in health food: Metabolism of quercetin in vivo and its protective effect against arteriosclerosis. J. Pharmacol. Sci. 2011, 115, 466-470. [CrossRef] [PubMed]

14. Chekalina, N.; Burmak, Y.; Petrov, Y.; Borisova, Z.; Manusha, Y.; Kazakov, Y.; Kaidashev, I. Quercetin reduces the transcriptional activity of NF-kB in stable coronary artery disease. Indian. Heart J. 2018, 70, 593-597. [CrossRef]

15. Ishisaka, A.; Kawabata, K.; Miki, S.; Shiba, Y.; Minekawa, S.; Nishikawa, T.; Mukai, R.; Terao, J.; Kawai, Y. Mitochondrial dysfunction leads to deconjugation of quercetin glucuronides in inflammatory macrophages. PLoS ONE 2013, 8, e80843. [CrossRef]

16. Ishizawa, K.; Izawa-Ishizawa, Y.; Ohnishi, S.; Motobayashi, Y.; Kawazoe, K.; Hamano, S.; Tsuchiya, K.; Tomita, S.; Minakuchi, K.; Tamaki, T.; et al. Quercetin glucuronide inhibits cell migration and proliferation by platelet-derived growth factor in vascular smooth muscle cells. J. Pharmacol. Sci. 2009, 109, 257-264. [CrossRef]

17. Iademarco, M.F.; Barks, J.L.; Dean, D.C. Regulation of vascular cell adhesion molecule-1 expression by IL-4 and TNF- $\alpha$ in cultured endothelial cells. J. Clin. Invest. 1995, 95, 264-271. [CrossRef]

18. Marui, N.; Offermann, M.K.; Swerlick, R.; Kunsch, C.; Rosen, C.A.; Ahmad, M.; Alexander, R.W.; Medford, R.M. Vascular cell adhesion molecule-1 (VCAM-1) gene transcription and expression are regulated through an antioxidant-sensitive mechanism in human vascular endothelial cells. J. Clin. Invest. 1993, 92, 1866-1874. [CrossRef]

19. Le, N.T.; Takei, Y.; Izawa-Ishizawa, Y.; Heo, K.S.; Lee, H.; Smrcka, A.V.; Miller, B.L.; Ko, K.A.; Ture, S.; Morrell, C.; et al. Identification of activators of ERK5 transcriptional activity by high-throughput screening and the role of endothelial ERK5 in vasoprotective effects induced by statins and antimalarial agents. J. Immunol. 2014, 193, 3803-3815. [CrossRef] 
20. Patel, R.; Varghese, J.F.; Singh, R.P.; Yadav, U.C.S. Induction of endothelial dysfunction by oxidized low-density lipoproteins via downregulation of Erk-5/Mef2c/KLF2 signaling: Amelioration by fisetin. Biochimie 2019, 163, 152-162. [CrossRef]

21. Deng, Y.; Lei, T.; Li, H.; Mo, X.; Wang, Z.; Ou, H. ERK5/KLF2 activation is involved in the reducing effects of puerarin on monocyte adhesion to endothelial cells and atherosclerotic lesion in apolipoprotein E-deficient mice. Biochim. Biophys. Acta Mol. Basis Dis. 2018, 1864, 2590-2599. [CrossRef] [PubMed]

22. Lee, M.; Yun, S.; Lee, H.; Yang, J. Quercetin mitigates inflammatory responses induced by vascular endothelial growth factor in mouse retinal photoreceptor cells through suppression of nuclear factor kappa, B. Int. J. Mol. Sci. 2017, 18, 2497. [CrossRef] [PubMed]

23. Zhao, F.; Wang, X.; Liang, T.; Bao, D.; Wang, Y.; Du, Y.; Li, H.; Du, J.; Chen, A.; Fu, Z.; et al. Effect of hyperbaric oxygen on tissue damage and expression of adhesion molecules and $\mathrm{C} 3$ in a rat model of renal ischemia-reperfusion injury after kidney transplantation. Ann. Transplant. 2020, 25, e919385. [CrossRef]

24. Chen, Y.; Waqar, A.B.; Nishijima, K.; Ning, B.; Kitajima, S.; Matsuhisa, F.; Chen, L.; Liu, E.; Koike, T.; $\mathrm{Yu}, \mathrm{Y}$; ; et al. Macrophage-derived MMP-9 enhances the progression of atherosclerotic lesions and vascular calcification in transgenic rabbits. J. Cell Mol. Med. 2020, 24, 4261-4274. [CrossRef] [PubMed]

25. Pereira, S.C.; Parente, J.M.; Belo, V.A.; Mendes, A.S.; Gonzaga, N.A.; do Vale, G.T.; Ceron, C.S.; Tanus-Santos, J.E.; Tirapelli, C.R.; Castro, M.M.; et al. Quercetin decreases the activity of matrix metalloproteinase-2 and ameliorates vascular remodeling in renovascular hypertension. Atherosclerosis 2018, 270, 146-153. [CrossRef]

26. Huang, H.; Liao, D.; Dong, Y.; Pu, R. Effect of quercetin supplementation on plasma lipid profiles, blood pressure, and glucose levels: A systematic review and meta-analysis. Nutr. Rev. 2020, 78, 615-626. [CrossRef]

27. Guide for the Care and Use of Laboratory Animals; National Institute of Health: Washington, DC, USA, 2011.

28. Imanishi, M.; Izawa-Ishizawa, Y.; Sakurada, T.; Kohara, Y.; Horinouchi, Y.; Sairyo, E.; Zamami, Y.; Takechi, K.; Chuma, M.; Fukushima, K.; et al. Nitrosonifedipine, a photodegradation product of nifedipine, suppresses pharmacologically induced aortic aneurysm formation. Pharmacology 2018, 102, 287-299. [CrossRef]

29. Droguett, D.; Castillo, C.; Leiva, E.; Theoduloz, C.; Schmeda-Hirschmann, G.; Kemmerling, U. Efficacy of quercetin against chemically induced murine oral squamous cell carcinoma. Oncol. Lett. 2015, 10, 2432-2438. [CrossRef]

30. Toth, M.; Sohail, A.; Fridman, R. Assessment of gelatinases (MMP-2 and MMP-9) by gelatin zymography. Methods Mol. Biol. 2012, 878, 121-135. 